\title{
The Fourier-Kelvin Stellar Interferometer: A Concept for a Practical Interferometric Mission for Discovering and Investigating Extrasolar Giant Planets
}

\author{
D. J. Benford*, W. C. Danchi*, R. J. Allen ${ }^{\dagger}$, D. Deming*, D. Y. Gezari*, \\ M. Kuchner ${ }^{* *+}$, D. T. Leisawitz* , R. Linfield ${ }^{\S}$, R. Millan-Gabet ${ }^{\text {Il }}$, \\ J. D. Monnier", M. Mumma*, L. G. Mundy ${ }^{\dagger \dagger}$, C. Noecker ${ }^{\S}$, J. Rajagopal*, \\ S. A. Rinehart ${ }^{\ddagger \neq}$, S. Seager ${ }^{\S \S}$ and W. A. Traub ${ }^{* *}$ \\ *NASA / Goddard Space Flight Center \\ ${ }^{\dagger}$ Space Telescope Science Institute \\ ${ }^{* *}$ Harvard-Smithsonian Center for Astrophysics \\ $\stackrel{\ddagger}{\ddagger}$ Russell Fellow \\ $\S_{\text {Ball Aerospace }}$ \\ ${ }^{\text {II }}$ California Institute of Technology \\ "University of Michigan \\ ${ }^{\dagger \dagger}$ University of Maryland \\ \#RC / Goddard Space Flight Center \\ ${ }^{\S}$ Carnegie Institution of Washington
}

\begin{abstract}
The Fourier-Kelvin Stellar Interferometer (FKSI) is a mission concept for a nulling interferometer for the near-to-mid-infrared spectral region $(3-8 \mu \mathrm{m})$. FKSI is conceived as a scientific and technological precursor to TPF. The scientific emphasis of the mission is on the evolution of protostellar systems, from just after the collapse of the precursor molecular cloud core, through the formation of the disk surrounding the protostar, the formation of planets in the disk, and eventual dispersal of the disk material. FKSI will answer key questions about extrasolar planets:

- What are the characteristics of the known extrasolar giant planets?

- What are the characteristics of the extrasolar zodiacal clouds around nearby stars?

- Are there giant planets around classes of stars other than those already studied?

We present preliminary results of a detailed design study of the FKSI. Using a nulling interferometer configuration, the optical system consists of two $0.5 \mathrm{~m}$ telescopes on a $12.5 \mathrm{~m}$ boom feeding a MachZender beam combiner with a fiber wavefront error reducer to produce a $0.01 \%$ null of the central starlight. With this system, planets around nearby stars can be detected and characterized using a combination of spectral and spatial resolution.
\end{abstract}

\section{THE ROLE OF FKSI}

The Fourier-Kelvin Stellar Interferometer (FKSI) is envisioned as a practical interferometric system for discovering and investigating extrasolar giant planets. Its capability is designed to enable a significant near term step in planet finding and characterization as compared to other present planet studies. FKSI supports key NASA Strategic Plan 
objectives in the area of expolanet research, and will serve as a pathfinder for the Terrestrial Planet Finder (TPF) and Darwin mission. The key questions FKSI seeks to find answers to are:

1. What are the characteristics of the known extrasolar giant planets?

2. What are the characteristics of the extrasolar zodiacal clouds around nearby stars?

3. Are there giant planets around classes of stars other than those already studied?

To answer these questions, the FKSI mission will complete four science projects:

- Detect $>25$ extrasolar giant plants (based on conservative predictions)

- Characterize their atmospheres with $\lambda / \delta \lambda=20$ spectroscopy

- Observe secular changes in spectrum

- Observe orbit of the planet

- Estimate density of planet; to determine if rocky or gaseous

- Determine main constituents of atmospheres from broad spectral features

- Search for exo-planets around nearby stars (not planned for observations with TPF)

- Nearby M dwarfs (>30 such stars) at distances within $10 \mathrm{pc}$

- Nearby F, G, K giants and subgiants, luminosity classes III and IV (>50 stars) within $30 \mathrm{pc}$

- Study circumstellar material

- Exozodiacal dust cloud measurements around nearby stars

- Search for companion stars

- Resolve debris disks, looking for clumpiness due to planets

- See star formation at high angular resolution

- Taxonomy of the evolution of circumstellar disks

- Characterize morphology, gaps, rings, etc.

There are currently more than 100 known extrasolar giant planets [1]. Because the discovery method uses only the light of the parent star, almost nothing is known observationally about the planets' atmospheres. The biggest surprise in this list is that there are many giant planets very close to their stars. Is this a bias of the radial velocity technique, or due to migration of the planet inward? FKSI will provide an important test of the bias of this observing technique.

More than 25 extrasolar giant planets are detectable with FKSI, and spectroscopy is possible for about half of these. A broad survey of nearby (K, M, class IV and III) stars is also possible. These studies will provide a firm observational foundation for the new field of exoplanetary science.

\section{FKSI MISSION CONCEPT}

FKSI is a two-element nulling interferometer (figure 1). The two telescopes, separated by $12.5 \mathrm{~m}$, are precisely targeted (by small steering mirrors) on the target start. The two path lengths are accurately controlled to be precisely the same. A phase shifter / 


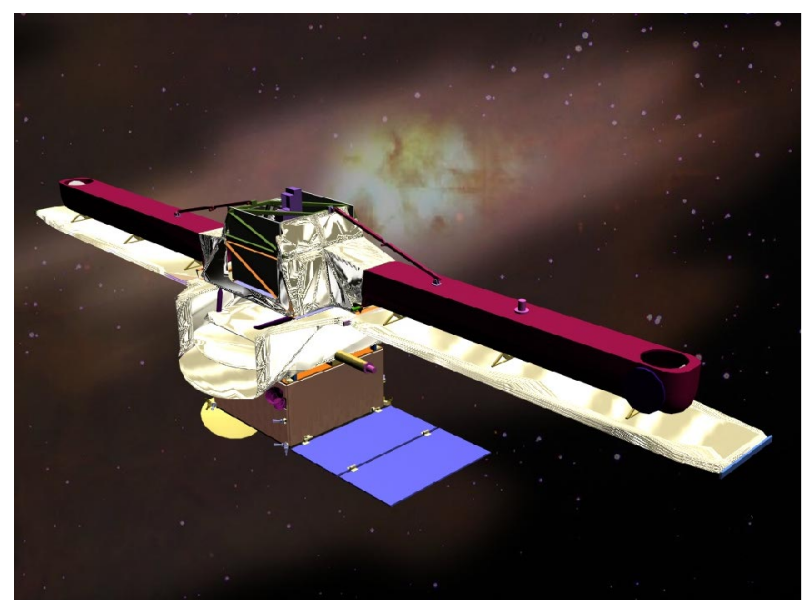

FIGURE 1. Artist's rendition of FKSI in operation.

beam combiner (via Mach-Zender interferometer) produces an output beam consisting of the nulled sum of the planet's light and the star's light. When properly oriented, the starlight is nulled by a factor of $10^{-4}$, and the planet light is undimmed. Accurate stellar spectroscopy is used to subtract this residual starlight, permitting the detection of planets much fainter than the host star, and at distances less than the $\sim 0.005^{\prime \prime}$ required for a similar imaging interferometer.

\section{FINDING PLANETS WITH FKSI}

FKSI's sensitivity is sufficient to detect and characterize extrasolar giant planets within $\sim 10$ pc. FKSI is comparable (to within an order of magnitude) in sensitivity to other missions in the $\simeq 5 \mu \mathrm{m}$ range (SIRTF, JWST), and superior to ground-based facilities. This is illustrated in the figure below, adapted from [2]. However, without a nuller, SIRTF and JWST will be hard pressed to extract the faint planet signal from the overwhelming star signal. As a result, FKSI will be a more capable planet finding instrument than other $\simeq 5 \mu \mathrm{m}$ systems.

We have simulated the response of FKSI to a planet and nulled star as the spacecraft rotates about its axis [3]. At a single wavelength, the intensity varies as a function of this rotation angle, as shown in figure 3 . Ratios of various wavelengths produce the unmodulated spectrum of the star, and the modulated spectrum of the planet. After rotating FKSI several times, a high signal-to-noise spectrum of the planet and star are obtained. The spectrum of the planet tells us about the atmospheric composition, mass, and age of the planet. The nature of the modulation pattern of the planet spectrum reveals the orbit of the planet, resolving the $M \sin i$ ambiguity present in radial velocity measurements. Watching this orbit varying over time will permit the study of the atmosphere of the planet as a function of its distance to its sun. 


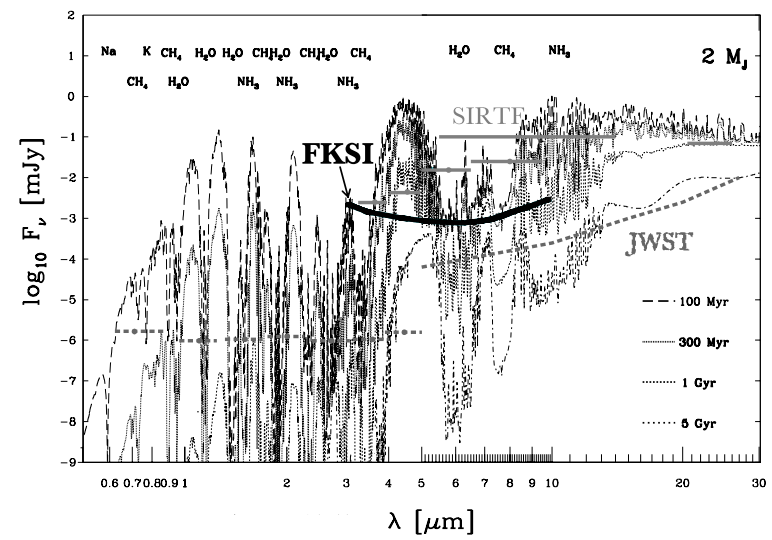

FIGURE 2. Predicted flux density of a $2 M_{J}$ planet as a function of age, at visible through mid-IR wavelengths, at a distance of $10 \mathrm{pc}$. FKSI can detect young giant planets of this sort with ease.
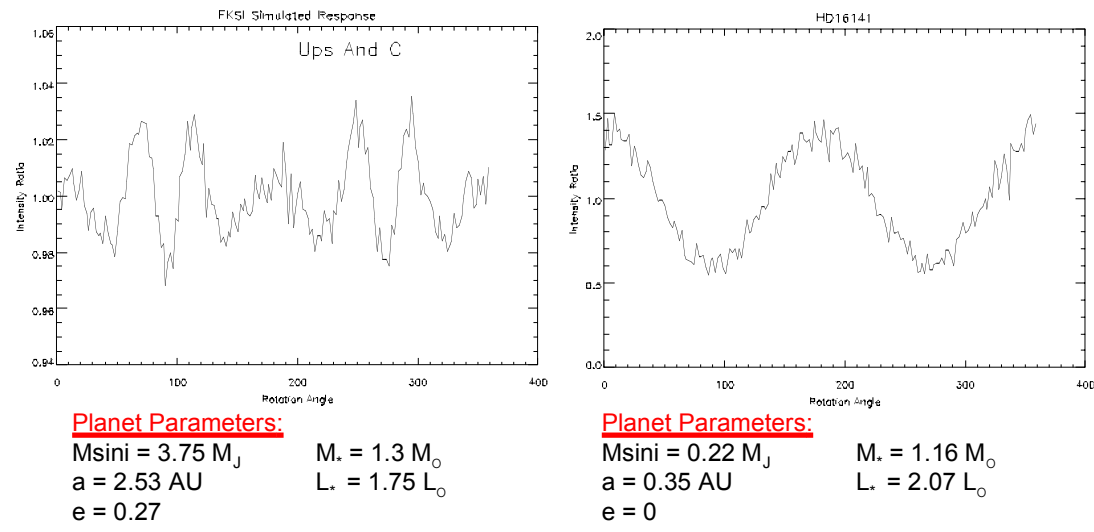

FIGURE 3. Simulation of FKSI response at a single wavelength to two simple planetary systems.

\section{ACKNOWLEDGMENTS}

We thank the FKSI engineering team at NASA/GSFC for their excellent work in producing the detailed design of the FKSI mission.

\section{REFERENCES}

1. Marcy, G.W., Butler, R.P., Fischer, D.A. and Vogt, S.S., in Scientific Frontiers in Research on Extrasolar Planets, ASP Conference Series, V.294, edited by D. Deming and S. Seager, pp.1-16 (2003)

2. Burrows, A., Sudarsky, D. and Lunine, J.I., ApJ, 596, pp.587-596 (2003)

3. Danchi, W.C., Deming, D., Kuchner, M.J. and Seager, S., ApJL, 597, pp. 57-60 (2003) 